\title{
Molecular Method in the Biocybernetics Clavitherapy, Non Invasive, Neuropathic and Oncology
}

\author{
Centrum Klawiterapii* \\ Department of Neurooncology, USA
}

*Corresponding author: Centrum Klawiterapii, Department of Neurooncology, USA.

\section{Introduction}

Clavitherapy is a new diagnostic and therapeutic direction in Reflexology. The method was discovered in the sixties of XX century. Polish psychologist doctor Ferdynand Barbasiewicz is the father of this pioneer methods. Mechanisms of Clavitherapy have scientific basis. To use this method in diagnosis and therapy, detail knowledge of anatomy of human nervous system, circulatory system and neurology is mandatory. Scientific basis of this method differs it from acupunctures or acupressure. Diagnostic and therapeutic procedures of Claviterapy are noninvasive and to date there is no contraindication to this method except: severe long-lasting under-nutritional conditions of patient susceptible to enzymic and metabolic collapse as well as patients with cardiac pace-maker and after organ transplantation.. From the observation of the results of Clavitherapy in patients it becomes clear that the method is highly effective in the treatment of nervous system disorders among others: all kinds of pain - headache, rheumatoid pains, lumbago; vasomotor disorders like migraine, cold limbs; demyelinization neuropathy; polineuropathy; multiple sclerosis (MS), ischemic brain strokes; parkinsonism; functional disorders after neurosurgery, after accidents; movement disorders, defects like scoliosis. Promising results have been observed in the treatment of sclerosis lateralis amyotrophica, personality disorders, neurosis and in different infectious diseases.

Human organisms, animals and plants have ability of selfregulations of their function, self-restoration to health and sometimes self-regeneration of sick of even lost organs. Within thousands years of human existence on the earth mankind discovered many methods to help sick organism in fighting against diseases. At present widespread diagnostic and therapeutic methods are available like: official medicine, homeopathy, ayurveda, iridology, acupuncture, acupressure, herbal medicine, quackery, magic.... Even now we are ignorant of mechanisms how homeopathic drugs do really work! though homeopathy is officially recognized, there is no laboratory proof of the therapeutic mechanisms of homeopathic drugs, but it does not overthrow however the theoretical foundation of homeopathic method.
Some thousands years ago in China specific therapeutic system was constructed for the treatment of different illnesses by direct influence onto skin. In China it was named "medicine of fire and metal" meanwhile in Europe just in XIX - century the matter of skinvisceral dependence in treatment of diseases of internal organs was taken in account and named this "acupuncture." This method works by needle sting of "points of acupuncture." In present day on the basis of acupuncture skin is treated by low temperature, electromagnetic field, acoustic waves, laser rays, electric currents, irritating substances etc. "Acupuncture" has been describing more and more new points on skin surface, which answer individual internal organs. Just such searches of new sensitive points of acupuncture on skin of man convinced the Polish scholar doctor Ferdynanda Barbasiewicza to brilliant conclusion about "Total Acupuncture" that is Clavitherapy. In this method in case of severe illnesses whole skin is submitted to the influence of clavics gradually keeping precise algorithm and network of bio-cybernetic functions.

\section{What is Reflexology}

Reflex is a Latin word and it means involuntary action. Reflexotherapyis a part of medical reflexology. The notion "medicine" contains elements of diagnosis and therapy. Reflexotherapy is the method where treatment /cure/healing are based on nerve reflexes and their effects. Nerve reflex is the involuntary action of the motor nerves under a stimulus from the sensory nerve and it emerges in effect of irritating nerve receptor located in skin and mucous membranes - dermatom, muscles - miotom, skeleton -sklerotom and directly nerves - neurotom. This knowledge is very useful, because it allows to steer such spontaneous features of organisms, as self-regulation, self-restoration to health, regeneration. Each living organism owns these features, saying from there "till life, there is genuine hope." This is a rational "saying". Ability to utilize reflexology to steer the effects of reflexes gives unlimited capability to steer functions of organisms. And this is the dream of ambitious physicians to have direct personnel influence on function of the patient's organism in a planned manner without any harmful sideeffects, observed very often during pharmacotherapy. 
Reflex therapy allows to approach the central nervous system indicating the sick organs which need cure or regulation of their functions, leaving at the hand of nature the recognition how deep and intensive should be the initiated curing process. In order to get therapeutic effect of reflex, contrary to present medical practice there is no need to carry detailed clinical or laboratory reconnaissance, to which physicians applying pharmacotherapy attach extra importance

Applying reflexotherapy in human or animal, we have impact on :

- $\quad$ regulation of vegetative process through inhibiting and prompting the function;

- $\quad$ inflammations, through vasomotor reactions initiated by reflexes;

- infection, through improvement of operational functions of nonspecific immune mechanism;

- pain, through increase of mainly endorphin concentrations;

- $\quad$ sleep disturbances dream and excessive psychomotor stimulation or depression, through boost of endorphin concentrations;

- $\quad$ scoliosis and spastic disturbances through the influence on regulation of skeletal, muscle tensions.

Answering the question like : what are the real mechanisms of healing process in reflexotherapy we can mention only hypothesis now, and this hypothesis needs researches to describe the actual natural causes of healing.

The first step in diagnosis and therapeutic procedure of Clavitherapy is "preliminary abreaction" on the skin of head being the primary stage of diagnosis and/or of therapy. Next step is stratified order of restoring of efficiency using whole surface of body. The therapy is carried on by clavics ( Lat. clavus - nail) on surface of skin, above anatomy of nervous system as well as on zones and biological active points including in this also acupuncture points. Clavitherapy generates by stimulation of neuro-physiologicaldermo-visceral reflex onto group of receptors: dermatom, further across neurochemical mediators/transmitters generates biocybernetic control/steering of neurotom, myotom and sclerotom. Through the mediation of nervous system it possible to steer repairing organism on sub-cellular endogenic level. Described process of bio-cybernetic steering through nervous system permits precisely complemental regulation of : cytokins, complements, interferons, endorfins and other endogenic factors to which other known therapeutic methods still do not have repairing access.

Clavitherapy is distinguished from other methods of reflexology by:

- It deals with the whole body not only with biologically active points.

- introduces the procedure of initial abreaction on the head as a prelude of diagnosis or therapy.

- $\quad$ adds pathologically changed zones on the skin (dandruff, algo-dystrophic zones, morphologically changed zones non- staining after stimulations, stained zones distinguished from the surroundings but unchanged morphologically, and other changes on skin described in detail in other chapters useful in therapy.

- $\quad$ creates and uses the unique system of bio-cybernetic dependence between the quality of procedure and feelings of the patients evoked by the procedure. This system allows, to continue the therapy always optimum for the present requirement of the patient.

- $\quad$ introduces due to bio-cybernetic dependence, stratified order of restoring of efficiency using whole surface of body

Klawiki Clavus is a Latin word, it means nail, is a very simple instrument made of surgical steel acid and fire proof and of 120 $\mathrm{mm}$ in length $4 \mathrm{~mm}$ in diameter. one end is conically shaped sharp enough and the other end is blunt two-sided phased. The blunt end resembles a screw-driver. It makes impression outwardly, as if lateral edges were produce easily, „, blasted “. This instrument in middle part, in one third length, is narrowest by a half and here total diameter is $2 \mathrm{~mm}$ ( look photo in atlas ). Besides, this part is strewed with sand the narrowest part is rough. Conical end, sharply ended serves for stimulating receptors from the group of dermatom, points and effective zones biologically active. It is possible to use in nonprofessional purpose kebabs know wooden sticks with one sharp end. During stimulation - on surface of skin - power of push, e.g. on head, is moderate - easy. It is obliged not to break continuity of epidermis. Obviously, in places of vast tissue e.g. buttock- somewhat it is necessary to press somewhat strongly. However, there, where the tissue is thin e.g. over bones or over visible blood vessels. it is necessary to press easily and cautiously.

On top of head clavics applied vertically act on skin by their own weight, enduring nerve tensions( cortical and central) in brain central, restoring patency of energy channels (acupuncture meridians) . It is necessary to adjust power of push, when we use single clavic, two , three and more ( e.g. seven ). A single clavic should be applied single delicately - cautiously , but it is possible to stimulate seven soundly. It is necessary to remember about power of push always, because ends are enough sharp and continuity of epidermus is easy to break particularly there, where zones are ischemic and stand from a long time. It is necessary to lay clavics in clusters e.g. 7 clavics.

Clavics laid in cluster (6-7 in number) first of all, are applied in detailed abreaction of the surface of skin. This type of stimulation are also applied on zones also biologically active. Zones discovered by F. Barbasiewicz like: ischemic zone, dansruff skin zone without inflammation, scars, folds, wrinkles, naevus, papilloma, russet and white spots, points and other zone it described in other places of this book. Different amounts of clavics (1-7) are used in abreaction depending on requirements -. It is wise to use more clavics in first day of procedures especially in individual patient with higher susceptibility to pain. In patients with low tolerance of pain initial stimulations should be performed with limited power of push. When oversensitivity on pain and burning sensations pass after several first days, we can stimulate in a faster rate, with more sound pression. It is necessary to control equality of sharp ends of clavics in cluster, otherwise the procedure will intensity the feeling of pain. Carelessly used clavics may disrupt continuity of epidermus. 
Speed of stimulation is an important factor of clavitherapy, the therapist is obliged to adjust it individually. It is obligatory to proceed particularly in first days of particularly very cautiously, adapting speed and power of push to physiological, physiological and nerve tolerance of pain , as at some persons influenced by reflex experiences of pains and burning sensation can cause feebleness even syncope. Under this situation the therapy procedure should be executed in lying position and control physiological, nerve and psychic form of the patient in order to avoid excessive tiredness of the patient. Sometimes it is necessary to use breaks for rest, and in longer procedures at every 3-4 hourly patient should take food. Biologically active points are stimulated by one, two or three clavics also for regulation and excitation of lost nerve potential, where they are receded or in zone of important plexus of nerve fibers where lack of conduction of nerve signal is diagnosed.

Other end of the clavic - blunt is applied when central abreaction was not achieved by sharp end and when we deal with sickness acute or chronic resistant to drugs.. Easy is executed on the surface of skin of head is executed easy cerebration in concrete topographic zone or brain centers particularly relentless , for abolishing cortical and central tensions in brain. The technique of cerebration is as following: 3 clavics are applied perpendicularly on the surface of the skin of the head, held, between thumb and indicative and middle fingers. Then the procedure is executed by three rotation left and right within a second. Cerebration is performed by easy movement in one place for several seconds. Procedures are executed after earliest abreaction of whole nervous system i.e. brain cortex, brain centers, peripheral nerves, efferent and afferent nerve of morphological and anatomical zones of sickness. And after regulation of personal nerve potential - and after excitation of conduction of nerve signal. The cerebration process has to be applied every day, in order to cure acute and chronic sickness also in zone of secondary change and other pathology, but not longer than for 10-14 days two times with a gap of 7 days.

Second blunt end of the clavic is also used in clavipressure. It is possible to add vaseline or cream to the blunt end of the clavic and press in zones shown in this book with moderate push using small round movement till the appearance of border of moderate pain, prompt biologically active points, shown in this book as algorithms of individual sicknesses for ceasation of pain and improvement of health.

Usually clavipressure is applied in children upto 7 years old. This form of cure is less painful, but at least it requires twice longer time for treatment and improvement.

\section{Critical Approach to Some Methods of Reflexotherapy}

In this place it has become necessary to approach critically to some seemingly natural methods of stimulations, as electrostimulation, elektropuncture, pharmacopuncture, laserpuncture, acupuncture, magnetotherapy and others. End of fifties of XX century the American scientists had carried careful research on stimulation of biologically active points on human skin by low power current electric of constant value. These points were stimulated to induce therapeutic changes in different functional disorders, particularly when attenuation of personal nerve potential takes place, in receded conduction of nerve signal in nerve fibers, in psychosomatic disturbances and infections. The research was carried to check variability of psychogalvanic ratio as a reaction to emotional, ambivalent experiences.

As it was concluded that such kind of electrostimulations are extremely harmful, because dangerous physiological disturbances in neurochemical, in hormonal and enzymatic information and others at endogenous, molecular, and submolecular levels take place. Productions of cytokins, complements, interferons, endorphins and other endogenous - less known factors in very wide morphological zones are disturbed. Some elements and structural factors undergo paralysis -at endogenous subcellular levels and they perish. It concerns in particularly permanent and irreversible disturbances in nerve cells in synapses of intercellular connection and magnetic field of cells. Information about steering of life, productions and distributions of endogenous factors on subcellular level and submolecular steering in blood are disrupted.

Therefore, fascination about electrostimulation electropuncture is incomprehensible. Probably, people who practice electropuncture are willing to bring about the renaissance of this method. They do not know the American research about harmfulness of electrostimulation in biocybernetic communications in receptor neurophysiology, mediators, regulations of life and divisions of cells in all endogenous levels, particularly processes of psychogenic, endogenous, neurophysiological and chemical selfregulation. Similar dart is applied in the case of electrostimulation, in neurological practices peroneal stimulator and other types destroying physiological life in cells and nerve fibers, particularly there, where susceptibility to enzymatic and metabolic collapse takes a stand for the reason of neurophysiological and ischemic disturbances.

Very harmful in its effect is pharmacopuncture ( application by injection of novocaine or similar chemical preparations in small doses to biologically active points) evokes damage of receptor and stimulating reflex apparatus, it disturbs subtile chemistry in stimulated zone). Similar things happen in case of acupuncture, where every time, there are mechanical damages of structure of points ,receptors etc by needles. Very often because of not enough precise hitting to biologically active points, they become physically and permanently broken down. All kind of blockades are harmful in acute and chronic neuralgia.

\section{Neoplasms}

The earliest cancer diagnosis AND THEIR ELIMINATION by OWN antibodies within a FEW DAYS. A special, personal satisfaction obtained from the discovery of the associations in the area of warts, brown spots of itching, pain, irritating the nervous system is a noticeable movement in the early stages of cancer. It shows people that In these areas of local circulation is lost. Cancer markers, cancerigenic/carcinogenic proteins take Places in the Morphological Structures and in the neighborhood, Preventing a smooth blood circulation. Therefore, the blood does not contain the appropriate values of endogenous Nutrients and selectively Appropriate antibodies, Which Are Able To Eliminate problems early infectious like viral, bacterial, fungi, or an excess concentration of markers, carcinogenic proteins. 
How clavitherapy methods can diagnose metastatic cancer in the earliest development?

Please take in your hand between the fingers two well-known clean toothpick and touch 2-3 mm from the warts, brown spots, etc.. changes to press the skin perpendicularly, non-invasively, with moderate force. If you experience around these lesions, or in a place from a particular distance from the change a strong and pervasive pain or burning sensation it means that you are at risk of malignancy at the earliest stage of development. Watch around on the skin or painful area from the skin surface $20 \mathrm{~cm}$ distant, do you observe somewhere in the neighborhood is different papilloma or brown spot skin changes, etc..? If the line between the skin lesions at some point crosses the spine May Be The Beginning of the paralysis and the local spinal pain.

Please take a sheet of A4 paper and put carefully applied on the longest side edge of the sheet from the pain, so that it adheres to the configuration of the body surface from the axis of the papilloma in a straight line to another near the papilloma , or brown spots. If you precisely stimulate on the line at the edge of a sheet of paper in turn the between these objects and the pain will linger for permeating from burning strictly in a straight line it means that it is very early onset of metastasis. If you go $1 \mathrm{~mm}$ to the side of a line between the objects there will not be any pain. Early metastasis is not necessarily merely a dermatome, skin. It may also take place inside the deeper anatomical structures. If the result of stimulation is burning sensation and pain in the first and second time lasts for about 3 seconds it means that the line runs in the dermatome of metastasis in the skin and connective tissue. Conversely, if the burning and pain revealed only after 2-4 seconds, and lasts longer than 3 seconds it means that the metastasis is located far deeper. Therefore, you need toothpicks to hold the pressure until the cessation of burning and pain. It should be for 5-7 days (2-3 times a day to stimulate around the lesion and carefully in a straight line between those lesions, then mute the pain in order to induce diligent congestion. This easy method confirms the diagnosis of intramuscular metastasis in a straight line between these skin changes or backbone. Metastasis may have a more complex character may be more numerous among those lesions, skin changes.

Clavitherapy allows to establish effective diagnosis of the disorder at the earliest stages -induced cancer and paralysis of the nervous system caused by these changes. Clavitherapy after careful restoration of circulation allows highly efficient antibodies to eliminate the local concentration of tumor markers (carcinogenic proteins) that this prevents the natural lines of ischemia and allows their own antibodies to appear. And also by stimulation of biologically active zones of the lymphatic system and activation of individual clusters corresponding lymph nodes and via X cranial nerve to stimulation of the ear edge for the precise activation of the relevant lymphatic system, local lymph nodes responsible are described in detail in the latest edition of the book and atlas of Clavitherapy.

Moreover, It Is necessary to eliminate harmful biogeologic radiation (as water veins, geopathic grid elements, various turbulence, the intensity of electro-magnetic field or infrasound, ultrasound, etc...) at home, school, workplace , breeding farms more information in the article of prof. dr. Ing Andrew Colic www. klawiterapia.com on my website.
Papilloma, naevus and russet spots require careful treatment and should not be punctured directly. They can present, as we know, form of tumor mutation, particularly melanoma malignum. It happens that some papillomas, naevus and russet spots are neutral, as if inoperative. But there are imaginary states of (conditions of) lack of activities. There are also papillomas, naevus and russet spots informatically active, which inform us about extensive different disturbances of physiological functions in human organism as it happens in case of cancers with metastasis. It concerns central particularly peripheral nervous system. It is necessary to know, that papillomae, naevus and russet spots render strong functional disturbances paralysis of nerve fibers, paresis and similar disturbances.

Active papillomae and russet spots successively limit blood supply, annex and evoke pathology of more and more area around them. Progressive ischemic process around them after a certain period lead to growth and concentration of cancerogenic protein called markers. One single papilloma or russet spot by evoking pathology of surrounding tissue can "create" new papilloma and russet spots, steer biocybenatically in straight line their growth and functions and after some time creating new aggressive papillomae and russet spots like cancer metastasis. These structure can also paralyze the physiological functions of nerve and internal organs.

NOTE! State when around papillomae and russet spots and among these new creatures in straight line during clavic stimulation strong penetrating pain and burning sensations are perceived, this is situation, which qualifies to liquidation of threat of malignant tumor by clavitherapy. Zones around active papillomae and russet spots are pressed without violation of the continuity of epithelium 2-3 $\mathrm{mm}$ from the papilloma or russet spots. The line of metastasis between these creatures on skin surface. It is proper to know, that pain that appear in straight line between these creatures indicate the direction of metastasis.When we go diverted with stimulation from straight line even for disappears.

It is indicated to execute these procedures very carefully three times a day, for receding pain and burning sensations days for 5-7 days pressing several times for strong induction of hyperaemia. This hyperaemia drives with blood to papillomae and russet spots antibodies, macrophages-killer cells and they forcefully destroy white cancerogenic proteins - markers. At the same time therapeutic points and zones and lymph nodes have to be stimulated for reconstruction of immune system (chapter IX, clavitherapy). During the procedures it is indicated to take 5 small meals with vitamin $C$ in the form of vegetables. It is proper to know, that human organism does not produce vitamin C. If no pain and burning sensations appear through successively 3-5 days the surgeon can delete these creatures without risk of activation and metastasis.

Another cause of neoplasm formation is demyelination neuropathy. Please, imagine the situation of a modern city without continuous supply of electricity, same thing happen in tissues when there is interruption of nerve conduction.

Tumor emerges in zone glutted with different kinds of metabolites, products of combustion, toxins with tendency to metabolic collapse. Papillomae and russet spots are information thrown outside on skin surface by the organism. Frequently 
very interesting cases take place, when already after first careful abreaction of papilloma and russet spots ( evoking hyperaemia) motor apparatus , e.g. lower extremities recover full proficiency recovers, abolishing of pains etc. , Chinese experts perform cerebration by left and right movements and introduce invasive needles under papilloma at an angle of $45^{\circ}$ during acupuncture that causes abolishing of information, neurophysiological, enzymatic and antibody disturbances, first of all macrophage disorders and this procedure evokes hyperaemia and destruction of cancergenic protein- markers. It happens, that after several abreactions and strong hyperaemia the papilloma becomes reduced or it disappears completely or falls off of the skin, level of individual kind of markers distinctly goes down to norm for peripheral blood.

\section{Acknowledgement}

None.

\section{Conflict of Interest}

No conflict of interest. 